\title{
A novel recombinant protein vaccine containing the different E7 proteins of the HPV16, 18, 6, 11 E7 linked to the HIV-1 Tat (47-57) improve cytotoxic immune responses
}

Tahoora Mousavi ( $\sim$ stm.jmums@gmail.com )

Mazandaran, Iran https://orcid.org/0000-0003-2505-370X

Reza Valadan

mazandaran, Iran

Alireza Rafiei

mazandaran, Iran

Ali Abbasi

mazandaran, Iran

Mohammad Reza Haghshenas

mazandaran, Iran

\section{Review}

Keywords: Fusion protein, E7, HPV vaccine, Therapeutic immunization, HIV1-Tat (47-57)

Posted Date: February 19th, 2021

DOI: https://doi.org/10.21203/rs.3.rs-195613/v1

License: (c) (1) This work is licensed under a Creative Commons Attribution 4.0 International License.

Read Full License

Version of Record: A version of this preprint was published at Biotechnology Letters on July 27th, 2021. See the published version at https://doi.org/10.1007/s10529-021-03166-2. 


\section{Abstract}

Objectives Human papillomavirus infection (HPV) is the most common viral infection which is causes of cervical, penal, vulvar, anal and, oropharyngeal cancer. E7 protein of HPV is a suitable target for induction of $T$ cell responses and controlling HPV-related cancer. The aim of the current study was to designed and evaluated a novel fusion protein containing the different E7 proteins of the HPV 16, 18, 6 and 11, linked to the cell-penetrating peptide HIV-1 Tat 49-57, in order to improve cytotoxic immune responses in in-vitro and in-vivo. Results In this study whole sequence of HPV16,18,6,11 E7-Tat (47-57) and HPV16,18,6,11 E7 cloned into the vector and expressed in E.coli (BL21). The purified protein was confirmed by SDS page and western blotting and then injected into the C57BL/ 6 mice. The efficiency of the fusion protein vaccine was assessed by antibody response assay, cytokine assay (IL-4 and IFN- $\gamma$ ), CD+8 cytotoxicity assay and tumor challenge experiment. Result showed that fusion proteins containing Adjuvant (IFA,CFA) could express higher titer of antibody. Also, we showed that vaccination with E7-Tat and, E7-Tat-ADJ induced high frequencies of E7-specific CD8+ T cells and CD107a expression as well as IFN-y level and enhanced long-term survival in the therapeutic animal models. Conclusion Our finding suggested that this novel fusion protein vaccine was able to induce therapeutic efficacy and immunogenicity by improving CD8+ T cell in TC-1 tumor bearing mice; so this vaccine may be appreciated for research against HPV and tumor immunotherapies.

\section{Introduction}

Human papillomavirus infection (HPV) is the most common viral infection which is causes cancer or genital warts (Haghshenas, Mousavi et al. 2016). Based on the location of HPV lesions, various types of cancer such as cervix, anal, vagina, throat, vulva, penis, and breast could be developed. More than 200 types of HPV have been identified, of which 40 types are belong to genital areas (Haghshenas, Mousavi et al. 2017) (Mousavi, Rafiei et al. 2020). HPV types are divided into high and low risk groups. Low-risk types such as 6 and 11 lead to $99 \%$ of genital warts and laryngeal papillomatosis; while $70 \%$ of cancers are related to high-risk types, such as 16, 18 (Akhondnezhad, Haghshenas et al. 2018). The HPV virus belongs to papillomaviridae which is encoding two structural $(L 1, L 2)$ and nonstructural protein (E1, E2, E4, E5, E6, and E7). E6 oncoprotein, is a key factor in tumor progression, act as P53 suppressor through binding to its $\mathrm{C}$-terminal region. This protein is a transcription factor that controls cell proliferation by preventing the $G_{1}$ to $S$ phase (Gulati, Huang et al. 2018). E7 oncoprotein is a small nuclear protein with no enzymatic activity. This protein is bound to the hypophosphorylated retinoblastoma protein (PRb); in this situation, the cell cycle moves to the $S$ phase. Then, DNA synthesis and cell proliferation takes place (Münger, Androphy et al. 2018). Regarding the importance of E6 and E7 in induction and maintenance of cellular transformation, these early genes have been reported as suitable targets for designing anti-tumor vaccines and controlling HPV infection (Li, Chen et al. 2017) (Tang, Yin et al. 2012).

In particular, dendritic cells (DCs) are one of the most important APCs and play an important role in immune response against viral infections (Eagar and Miller 2019). DCs function, macrophageinflammatory protein-1a (MIP)-1a, MIP-1 $\beta$ and RANTES genes activation is associated with the high 
expression of MHC-I, CD40, CD80, CD86 and production of IL-1 $\beta$, TNF-a, IL-12, IL-15 cytokines (FanalesBelasio, Moretti et al. 2002). The serious problem associated with protein and peptide vaccines is that the structure of peptide antigen could be altered and do not elicit strong immune response (Du, Liang et al. 2020). On the other hand, these proteins enter the cells via an endocytic and activate the MHC-II presentation pathway; so they are unable to induce strong cytotoxic CD8+ T cell response (Blum, Wearsch et al. 2013). Today, there are several ways are used to transfer protein in to the cell. Cell penetrate peptides (CPP) such as Tat, Pep-1, LAL, Cady-2, P28, hpp10 peptide with potential interact to proteins and peptides are able to transfer different types of cargo (protein and peptides) into the cell (Shahbazi and Bolhassani 2018). It is suggested that a novel delivery system, Tat protein (small and soluble protein with 86-101 amino acids), with high efficiency and low cytotoxicity is able to transport peptides and proteins into cytoplasm via the plasma membrane and it is well-suited for the delivery of antigenic peptide to MHC-1 presentation pathway (Zou, Peng et al. 2017). Study shows that vaccination with Tat protein provides protection against viral replication, which can induce Th1 immune response as well as the Cytotoxic T lymphocytes (CTLs) response (Alipour, Mahdavi et al. 2017).

Prophylactic and therapeutic vaccination is highly effective in control and prevention of HPV infection. Two prophylactic HPV vaccines (Gardasil, Cervarix) are composed of L1 virus-like particles (VLPS) and they are used for the prevention of HPV infection and cervical cancer (Barra, Leone Roberti Maggiore et al. 2019). In the current study, we designed a novel therapeutic vaccine based on E7 protein of HPV16,18,6,11 and Tat peptide against cervical cancer. HPV16, 18, 6, 11 E7- Tat (47-57) protein was assessed in vitro and examined for induction of E7-specific humoral and cell-mediated immune responses in C57BL/6 mice model. This study shows how a novel fusion protein induce therapeutic efficacy against HPV16-positive tumor cells in mice.

\section{Material And Methods}

\subsection{Vaccine design and plasmid construction}

In brief, the nucleotide sequence of E7 genes from HPV6 (accession no. FR751338.1), HPV11 (accession no. JN644141.1), HPV18 (accession no. MF288727.1), and HPV16 (accession no. K02718.1) and Tat (YGRKKRRQRRR) were in silico assembled with appropriate linker (GGGS) using CLC Workbench 8.1. Whole sequence was synthesized by Biomatik, (Cambridge, Canada) and cloned into His-tag harboring pET-22b vector. In order to establish an additional fusion protein by the lack of Tat sequence, Nco1 and Xho1 restriction sites were placed between the chief protein (E7-TAT) and then cloned to pET-28a vector. The plasmids pET22b-E7-TAT and pET28a-E7 were purified.

\subsection{Production of fusion proteins (E7-TAT, E7)}

Expression plasmids (pET-22b , pET-28a) were transformed to the E.coli (BL21) and cultured on LB broth containing specific antibiotics, and then incubated at $37^{\circ} \mathrm{C}$ by shaking $170 \mathrm{rpm}$. The OD was checked until the optimal density reached to $0.6-0.8$ at $600 \mathrm{~nm}$. One milliliter of the culture without inducer was used as a negative control. The culture was induced by adding 0.1-0.5 mM of Isopropyl-D-thiogalactoside 
(IPTG) and incubated at $37^{\circ} \mathrm{C}$ for $16 \mathrm{~h}$. Harvest bacterial cells were centrifuged at $6000 \mathrm{~g}$ at $4^{\circ} \mathrm{C}$ for 15 min; then the supernatant discarded and cell pellet frozen at $-20^{\circ} \mathrm{C}$. After re-suspending pellet in the buffer solution, it is found that the expressed proteins aggregated in inclusion bodies. The next day bacteria were re-suspended in buffer [ $50 \mathrm{mM}$ Tris-HCL PH=7.5, 1.5M NaCl, 20mM Imidazol, Urea 8M, Guanidine $6 \mathrm{M}, 5 \mathrm{mM} 2 \mathrm{ME}$ and $2 \%$ Triton $100 \mathrm{x}$ ]. Then cells were sonicated on ice for $3 \mathrm{~min}$ with $30 \mathrm{sec}$ interval. The mixture then centrifuged at $6000 \mathrm{~g}$ at $4^{\circ} \mathrm{C}$ for $15 \mathrm{~min}$ and the soluble fraction was transferred to a new $15 \mathrm{ml}$ tube. At the final, $500 \mathrm{mM}$ Imidazol and affinity chromatography based on Ni-NTA (Qiagen) column were used for purification of the recombinant fusion protein using 6 xHis-tag.

\subsection{SDS page and western blotting analysis}

After purification of recombinant fusion protein, dialysis was performed for the removal of residual salts, then concentration of protein was assessed by BCA kit (DNA Biotech, Iran) according to the manufacturer's instructions. After that, to confirm of the presence of purified protein, the fraction was analyzed by the $12 \%$ SDS-PAGE gels (w/v) and then transferred to a PVDF membrane for $2 \mathrm{~h}$ by Bio-Rad transfer system. PVDF membrane was shacked by TTBS (Tween $200.1 \%$, Tris base $1 \mathrm{M}, \mathrm{NaCl} 0.9 \%$ ) and $5 \%$ skimmed milk overnight. After washing with TTBS three times, the membrane treated with mouse anti-His-tag antibody (Thermo Fisher Scientific,US) at 1:1000 dilutions at room temperature (RT) by shacking $2 \mathrm{~h}$, and then washed with TTBS three times. The membrane was treated in the secondary HRPconjugated antibody (Thermo Fisher Scientific,US) at 1:5000 dilutions by shacking $2 \mathrm{~h}$ at RT; then it was washed with TTBS three times. The result was analyzed by staining with ECL and Luminescence Image System (Hansor, Taiwan).

\subsection{Cell culture}

TC-1 cells (expressing HPV16 E6 and E7 and generated by co-transformation of C57BL/6 mouse lung epithelial cells), KB cell (contain human papillomavirus18 (HPV18) and Caski cell (contain human papillomavirus16 (HPV16) sequences) were purchased from the Pasteur Institute of Iran. TC-1 cells were cultured in Dulbecco's modified Eagle's medium (DMEM) supplemented with $10 \%$ fetal bovine serum (FBS) and antibiotic in a humidified atmosphere of $5 \% \mathrm{C02}$. Caski and KB were cultured in Roswell Park Memorial Institute (RPMI) supplemented with $10 \%$ fetal bovine serum (FBS) and antibiotic in a humidified atmosphere of $5 \% \mathrm{CO} 2$.

\subsection{Preparation of cell lysate}

KB and Caski cells were De-attached and washed with PBS buffer for three times. The cells were centrifuged at $10000 \mathrm{~g}$ for $10 \mathrm{~min}$. After that the pellet were subjected to freeze-thaw between liquid nitrogen and $37^{\circ} \mathrm{C}$ water bath for 3-4 times. The amount of total protein was measured by BCA kit according to the manufacturer's instructions. In present study, HPV6 and HPV11 cell lysate were obtained from clinical sample.

\subsection{Mice immunization}


Inbred female C57BL/6 mice (8-12 weeks old) were purchased from the Pasteur Institute of Iran and maintained under specific pathogen free conditions. Six groups of five mice $(n=5)$ were subcutaneously injected with $20 \mu \mathrm{g} / \mathrm{ml} \mathrm{E7-Tat,} 20 \mu \mathrm{g} / \mathrm{ml}$ E7-Tat plus Freund's adjuvant (50: $50 \mathrm{v} / \mathrm{v}$ ), $20 \mu \mathrm{g} / \mathrm{ml}$ E7, 20 $\mu \mathrm{g} / \mathrm{ml}$ E7 plus Freund's adjuvant (50: $50 \mathrm{v} / \mathrm{v}$ ), PBS and Freund's adjuvant at the right flank three times on days 0,14 , and 28 . Complete Freund's adjuvant (CFA) and incomplete Freund's adjuvant (IFA) were emulsified with protein for the 1 st and 2 nd respectively. For analysis of specific $\lg G$ antibody assay, the blood samples were obtained before vaccination, two weeks after the second vaccination and one week after the last vaccination. (Table 1) (Fig 1).

\subsection{Antibody response assay}

Mice were sacrificed 10 days after the third immunization and the level of specific IgG antibody was measured by ELISA for detection of antibodies to E7 and E7-Tat. Briefly, $10 \mu \mathrm{g}$ of E7 and E7-Tat protein were coated with PBS buffer in 96 well flat-bottom ELISA plate (Nunc), overnight at $4^{\circ} \mathrm{C}$. After washing with PBS buffer and Tween 20 (5\%) for 3 times, wells were blocked with \% BSA (bovine serum albumin) for $2 \mathrm{~h}$ at $37^{\circ} \mathrm{C}$. Then, after washing, serum samples were diluted 1:100 to each well in triplicate and incubated for $1.5 \mathrm{~h}$ at RT. After washing, a goat anti-mouse IgG HRP antibody (diluted 1:10,000 in 1\% BSA/PBS-Tween) was added to each cell, and the plate was incubated for $1.5 \mathrm{~h}$ at RT. The plate was washed and TMB solution was added to each well. After $10 \mathrm{~min}$, the stop solution (H2SO4 (2N)) was added and the absorbance was measured at $450 \mathrm{~nm}$.

\subsection{MTT assay for toxicity of HPV6 and HPV16 cell lysate}

The MTT assay was used for evaluation of the toxicity of the clinical and cell lysate samples (HPV6 E7 and HPV16). Briefly, normal C57 BL/6 splenocytes (500,000/well in RPMI 1640 containing 10\% FBS) were seeded onto 96 -well culture plates in triplicate. After $24 \mathrm{~h}$ of culture, cells were stimulated with different doses of protein $(100 \mu \mathrm{g} / \mathrm{ml}, 50 \mu \mathrm{g} / \mathrm{ml}$, and $20 \mu \mathrm{g} / \mathrm{ml})$ and incubated for $48 \mathrm{~h}$. After incubation the supernatants were removed and the MTT ([3-(4,5-dimethylthiazol-2-yl)-2,5-diphenyltetrazoliumbromide], Sigma, $5 \mathrm{mg} / \mathrm{mL}$ in PBS) was added and then cells were incubated at $37^{\circ} \mathrm{C}$ in $5 \% \mathrm{CO} 2$ for $4-6 \mathrm{~h}$. The absorbance of purple formazan crystal was measured at $590 \mathrm{~nm}$ by an ELISA reader after dissolution in dimethyl sulfoxide (DMSO), Sigma.

\section{9 cytokine assay (IL-4 and IFN-Y)}

After sacrificing the mice, the spleen of each mice was isolated. A total of $\left(5 \times 10^{5} / \mathrm{ml}\right)$ splenocytes of mice in each treatment group were stimulated with $100 \mu \mathrm{g} / \mathrm{ml}$ concentrations (Kim, Hong et al. 2006) of HPV6, HPV11, KB (HPV18), Caski (HPV16) cell lysate and 10 $\mu / \mathrm{ml}$ of each purified protein (HPV E7 and HPV E7-Tat) in RPMI 1640 medium supplemented with $10 \%$ FBS and $100 \mu \mathrm{g}$ of streptomycin per ml for $48 \mathrm{~h}$ in a $37 \mathrm{C}^{\circ}, 5 \% \mathrm{CO} 2$ incubator. After that supernatant was collected for the presence of cytokines IL-4 and IFN- $\gamma$. Concentration of cytokines were determined by specific sandwich ELISA-Reader according to the manufacturer's instruction. (Invitrogen, United States) with C.N 88-7044 (Mouse IL-4 Uncoated ELISA) and 88-7314 (Mouse IFN gamma Uncoated ELISA). 


\subsection{CD+8 cytotoxicity}

Splenocytes from each mouse harvested in cell medium at $5 \times 10^{5} / \mathrm{ml}$ and stimulated with $100 \mu \mathrm{g} / \mathrm{ml}$ concentrations of HPV6, HPV11, KB, Caski cell lysate and $10 \mu / \mathrm{ml}$ of each purified protein (E7 and E7-Tat) for $48 \mathrm{~h}$ in a $37 \mathrm{C}^{\circ}, 5 \% \mathrm{CO} 2$ incubator. Then the pellet were collected and labeled with FITC-conjugated anti-CD8a and PE-conjugated anti-CD107a antibody (invitrogen, USA) for cytotoxicity activity. We used isotype matched antibodies as control (Invitrogen, USA). Data were analyzed using Flowmax software (TreeStar, San Carlos, CA).

\subsection{Tumor model and vaccination}

For therapeutic studies six groups of mice $(n=5)$ challenged with $3 \times 10^{5}$ TC-1 cells subcutaneously in the right flank. On day 6 post-tumor, mice were challenge with various formulation of vaccine containing 20 $\mu \mathrm{g} / \mathrm{ml}$ E7-Tat, $20 \mu \mathrm{g} / \mathrm{ml}$ E7-Tat plus Freund's adjuvant, $20 \mu \mathrm{g} / \mathrm{ml} \mathrm{E7,} 20 \mu \mathrm{g} / \mathrm{ml}$ E7 plus Freund's adjuvant, PBS and Freund's adjuvant on day 0, 14, 28 (Table 1). Following the tumor cells challenge, tumor length, width and depth was measured by manual calipers every week.

\subsection{Statistical Analysis.}

Statistical analysis was assessed using ANOVA (one-way and two-way) followed by Tukey's test (Graph Prism Pad version 8.0.1 soft-ware). Survival was analyzed using the log-rank (Mantel-Cox) test. This result considered statistically significant as a $P$ value of $<0.05$.

\section{Results}

\subsection{Identification of recombinant protein}

DNA fragments of the HPV 16,18,6,11 E7-Tat and the short TAT peptide insertion element, 'YGRKKRRQRRRS', were subcloned into the corresponding restriction sites of the $\mathrm{pET}-22 \mathrm{~b}$ plasmid. The construct of $p E T-22 b$ vector containing C-terminal HisTag and N-terminal Tat (47-57) (YGRKKRRQRRRS) is shown in Fig.2 (A,B). HPVE7-Tat (47-57) digested with Nco1 and Xho1 is shown in Fig. 3.

\subsection{Expression and purification of the recombinant fusion protein}

The plasmid pET22b E7-Tat vector was purified and transformed into BL21. Fusion protein expression was induced by adding IPTG to LB broth media. For optimization of the protein expression, some various conditions were tested, such as temperature, concentration of IPTG, induction time of expression and host. This study shows that induction with $0.1-0.5 \mathrm{mM} \mathrm{IPTG}$ at $37^{\circ} \mathrm{C}$ for $16 \mathrm{~h}$ is suitable condition for expression of the fusion protein (Fig.4.A,B). The sonicated cell supernatants were purified using Ni2+ (NiNTA superflow) affinity chromatography. Purification of HPVE7-Tat and HPVE7 protein with the molecular weight of 48.57 and $46.68 \mathrm{KDa}$ is shown in Fig. $5(\mathrm{~A}, \mathrm{~B})$. The purified recombinant protein transferred to PVDF membrane for western blotting analysis then, they were detected by anti-His antibody (Fig.6. A,B). According to the BCA protein assay kit, the total concentration of protein was calculated $600 / \mathrm{ml}$. 


\subsection{Protective response elicited in E7-Tat-ADJ group}

According to this point that specific neutralizing antibodies are effective in preventing of HPV infection (Wu, Ma et al. 2019), in this study the sera from each vaccinated group (E7-TAT-ADJ, E7-ADJ, E7, E7-TAT, PBS, ADJ) treated with $10 \mu \mathrm{g} / \mathrm{ml}$ of the HPV E7-Tat and HPV E7 protein for examination of specific lgG antibody production. Analysis of specific IgG response were assessed against the HPV E7-Tat and HPV E7 protein in various groups in the second and third immunization. Present data shows that antibody response against antigens is high in all vaccinated groups compared to control groups. Indeed, our data indicate that the highest specific IgG was observed in E7-TAT-ADJ groups among all groups after the second and third immunization (<0.0001).Briefly, our finding shows that the mice immunized with E7-TatADJ protein was able to stimulate strong IgG antibody with compared to other groups $(<0.0001)$. We suggest that Adjuvant in combination with Tat peptide can be effective at elevating the serum antibody level (Fig 7).

\subsection{The $100 \mu \mathrm{g}$ of cell lysate is the safe concentration for toxicity}

MTT was performed for measuring of toxicity effect of HPV16 and HPV6 cell lysates at different concentration. MTT assay shows that HPV16 and HPV6 cell lysates at ratio of $100 \mu \mathrm{g}$ did not induce toxic effects compared to $20 \mu \mathrm{g}$ and $50 \mu \mathrm{g}$ during $48 \mathrm{~h}$ period. It is interesting that there is no statistical difference was detected in toxicity rate between these groups (Fig 8).

\subsection{IFN- $Y$ production increased in E7-TAT-ADJ vaccinated groups}

As we know, IFN-y plays a critical role in enhancing the innate and adaptive immune responses against tumor growth. Data indicates that vaccination is an important mechanism for IFN-y producing. In this present study we examined the protective IFN- $\gamma$ response in vaccination groups. The splenocyte of all vaccinated groups were stimulated with HPV16, HPV18, HPV6, HPV11 cell lysates, E7-Tat and E7 protein in-vitro. All groups of mice immunization with different vaccine modalities increased the level of IFN- $Y$ secretion as compared to control groups $(p<0.05)$. The cytokine results shows that the level of IFN- $Y$ secretion in E7-TAT and E7-TAT-ADJ vaccinated groups were effectively increased in compared to other groups $(P<0.05)$ (Fig 9). Briefly, Among all groups the group immunized with the E7-TAT-ADJ showed a significant IFN-y response compared to other groups $(P<0.05)(F i g 9)$.

On the other hand, the splenocyte of all vaccinated groups were stimulated with HPV16, HPV18, HPV6, HPV11 cell lysates, HPV E7-Tat and HPV E7 protein and did not show IL-4 producing in any group ( $P>0.05$, data not shown).

\subsection{E7-TAT-ADJ induced anti-tumor T cell response}

Study shows that CD107a expressing and IFN-y producing CD8+ T cell are important immune response against TC-1 tumors (Kim, Kim et al. 2004); so we examined whether immunization with the novel vaccine could induce E7-specific cytotoxic activity. In the present study, two weeks after the third immunization, the splenocytes were harvested and the splenocytes of immunized animal were stimulated 
with E7-Tat, E7 protein and HPV16, HPV18, HPV6, HPV11 cell lysates. As shown in Fig 10, the frequency of CD107a expression in E7-TAT-ADJ vaccinated group was significantly higher than in the other groups. Our finding shows that the CTL response in the E7-TAT-ADJ immunized mice is highly effective and enhanced the antigen-specific immune response.

\subsection{Therapeutic immunization of tumor-bearing mice with E7-Tat-ADJ and E7-TAT promote overall survival}

In the therapeutic strategy, the mice were immunized with E7-Tat, E7-Tat-ADJ, E7, E7-ADJ, PBS and Adjuvant for analysis of protection against tumor development. The results indicated that anti-tumor immune responses elicited by E7-Tat-ADJ and E7-TAT (Fig.11).

For determination of survival, tumor-bearing mice were monitored 45 days. Fig. 12 shows that 100\%(5/5) of animals treated with PBS and ADJ were moribund by day 35. Our results indicate that survival of TC-1bearing mice treated with E7-Tat-ADJ and E7-TAT is significantly longer than other groups 60\% (3/5) over 45 day period and it could protect mice from tumor growth compared to E7 and E7-ADJ groups. more ever, in the result of current study, 100(5/5) of treated groups with E7 and E7-ADJ were moribund by day 40-41. Our finding suggest that it was statistically significant differences between all vaccinated groups $(P<0.0001)$.

\section{Discussion}

In the current study of therapeutic vaccines, the HPV16,18,6,11 E7-Tat and HPV16,18,6,11 E7 recombinant fusion protein expressed in E.coli with the highest growth and protein synthesis rate. HPVs types encode two non-structural E6 and E7 oncoproteins which induce E6/E7-specific T cells. E6 protein inactivate tumor suppressor protein P53 and E7 protein bind to pRb which is responsible for the maintenance of the cell cycle (Su, Xu et al. 2016). Our findings indicate that the recombinant fusion protein is able to induce cellular immune response and CTL activity. Similar to the present study, a review reported that DNA vaccination (VGX-3100) using E7 and E6 protein is able to improve cellular and humoral immune responses, IFN- $\gamma$ production and CD $8+T$ cell activation in patients with HPV 16/18-positive CIN2/3 in a phase 1 clinical study (15).

It is well known that APCs capture exogenous antigens through phagocytosis and different mechanisms. The exogenous antigens can be presented on MHC class II. The processing of exogenous antigens by cytosolic pathway can be present by MHC-I and induce the production of specific CD $8+\mathrm{T}$ cells (Mellman and Steinman 2001). There are several strategies to increase antigen cross-presentation pathways, including the use of gp96 (Singh-Jasuja, Toes et al. 2000), fusionic (Laus, Graddis et al. 2000), membrane protein A of Klebsiella pneumoniae (Jeannin, Renno et al. 2000), and cationic molecules. Tat fusion protein is safe and it is capable of binding antigens to the MHC-I and increases the production of antigen-specific CD8 + T cells; therefore it is used in cancer therapy, immunotherapy and therapeutic vaccines (Cafaro, Tripiciano et al. 2019). This present study indicate that the Tat peptide of HIV-1 is essential for potentiating the induction of cell-mediated immunity. A result of study demonstrated that 
cationic proteins such as HIV-Tat sequences, is able to plays an important role for transferring proteins and drugs (20-200KD) to the mammalian cells (Tanaka, Dowdy et al. 2003). Another finding suggested that, protein vaccination conjugated Tat protein is able to improve CTL, IFN- $\gamma$ and NK cells. Also it has been reported that E7 (49-57) Tat (49-57) nanoparticle plays an effective role to improve CD8 + T cellmediated antitumor immunity (Zhao, Wang et al. 2013).

Freund's adjuvants are known as a delivery system and can be used to enhance the anti-tumor cellular immune responses. These Adjuvants are used in protein vaccines and able to increase the production of innate cytokines such as IFN-y, IL-12, IL-18, and IL-15 which are activated NK cell and CD107a expression (Mousavi, Saravi et al. 2019). Freund's adjuvants alone are not insufficient for CD8 + T cell priming and tumor regression. In the present research, it was demonstrated that Freund's adjuvants with TAT peptide in E7-TAT-ADJ vaccinated groups are potent adjuvants in priming of immune response and antitumor activity and also increase the survival of tumor-bearing mice. It has been shown that vaccination with HPV-16 cVLP containing CFA (Freund's complete adjuvant) and IFA (Freund's incomplete adjuvant) enhances the production of IgG antibodies and suppresses tumor growth by production of tumor-specific CD4 + T cells (Monroy-García, Gómez-Lim et al. 2014). A research study indicated that immunization with L1, E6 and E7 protein plus CFA and IFA adjuvant is able to increases the prophylactic efficacy (Xu, Wang et al. 2016). Furthermore another findings suggested that vaccination with $E 7$ and L1 peptide combined with IFA adjuvant elicits a strong humoral and cellular immunity and increases the CD8 + T cell anti-tumor response and anti-HPV antibodies (Bates, Uematsu et al. 2009) (Levy, Goriely et al. 2013).

Three types of HPV vaccines including Gardasil, Cervarix and Gardasil 9 based on L1 protein are prophylactic and can induce long-term immune responses. Because of the importance of cervical cancer in community, many research have focused on various therapeutic vaccines. This present results showed that HPV16,18,6,11 E7-Tat recombinant fusion protein with Freund's adjuvant is a novel vaccine which is able to increase CTL response and improve specific antibody in animal model. This vaccine let to increase survival rate by improving CD8 + T cell; so it should be appreciate vaccine against HPV.

\section{Declarations}

Funding: This study was funded by Mazandaran University of Medical Sciences, Iran with grant No. 3049 and conducted in the Molecular and Cell Biology Research Center, Faculty of Medicine.

Conflict of interest: Author declares that no conflict of interest.

Ethical approval: All procedures performed in studies involving mice participants were in accordance with the ethical guideline of the International Council for Laboratory Animal Science (ICLAS) ( IR.MAZUMS.REC.96.3049). This article does not contain any studies with human participants performed by any of the authors.

\section{Author contributions}


Valadan Reza did most of the experiments and was a major contributor in writing the paper. Mousavi Tahoora and Abbasi Ali collected clinical tissues and performed the PCR assay. Rafiei Alireza performed the statistical analysis. Haghshenas Mohammad Reza designed the study and was also a major contributor in writing the paper. All authors read and approved the final manuscript.

\section{References}

Akhondnezhad, M., M. R. Haghshenas, M. Ghasemi and T. Mousavi (2018). "The prevalence and genotyping of human papillomavirus in patients with oral tumors in health centers and clinics of Mazandaran in Iran." VirusDisease 29(3): 297-302.

Alipour, S., A. Mahdavi and A. Abdoli (2017). "The effects of CpG-ODNs and Chitosan adjuvants on the elicitation of immune responses induced by the HIV-1-Tat-based candidate vaccines in mice." Pathogens and disease 75(2).

Barra, F., U. Leone Roberti Maggiore, G. Bogani, A. Ditto, M. Signorelli, F. Martinelli, V. Chiappa, D. Lorusso, F. Raspagliesi and S. Ferrero (2019). "New prophylactics human papilloma virus (HPV) vaccines against cervical cancer." Journal of Obstetrics and Gynaecology. 39(1): 1-10.

Bates, J. T., S. Uematsu, S. Akira and S. B. Mizel (2009). "Direct stimulation of tlr5+/+ CD 11c+ cells is necessary for the adjuvant activity of flagellin." The Journal of Immunology_ 182(12): 7539-7547.

Blum, J. S., P. A. Wearsch and P. Cresswell (2013). "Pathways of antigen processing." Annual review of immunology. 31: 443-473.

Cafaro, A., A. Tripiciano, O. Picconi, C. Sgadari, S. Moretti, S. Buttò, P. Monini and B. Ensoli (2019). "AntiTat Immunity in HIV-1 Infection: Effects of Naturally Occurring and Vaccine-Induced Antibodies Against Tat on the Course of the Disease." Vaccines 7(3): 99.

Du, R.-H., L.-R. Liang, C.-Q. Yang, W. Wang, T.-Z. Cao, M. Li, G.-Y. Guo, J. Du, C.-L. Zheng and Q. Zhu (2020). "Predictors of mortality for patients with COVID-19 pneumonia caused by SARS-CoV-2: a prospective cohort study." European Respiratory Journal 55(5).

Eagar, T. N. and S. D. Miller (2019). Helper T-cell subsets and control of the inflammatory response. Clinical Immunology_(Fifth Edition), Elsevier: 235-245. e231.

Fanales-Belasio, E., S. Moretti, F. Nappi, G. Barillari, F. Micheletti, A. Cafaro and B. Ensoli (2002). "Native HIV-1 Tat protein targets monocyte-derived dendritic cells and enhances their maturation, function, and antigen-specific T cell responses." The Journal of Immunology. 168(1): 197-206.

Gulati, T., C. Huang, F. Caramia, D. Raghu, P. J. Paul, R. J. Goode, S. P. Keam, S. G. Williams, S. Haupt and O. Kleifeld (2018). "Proteotranscriptomic measurements of E6-Associated Protein (E6AP) targets in DU145 prostate cancer cells." Molecular \& Cellular Proteomics: mcp. RA117. 000504. 
Haghshenas, M. R., T. Mousavi, M. Kheradmand, M. Afshari and M. Moosazadeh (2017). "Efficacy of human papillomavirus 11 protein vaccines (cervarix and gardasil) in reducing the risk of cervical intraepithelial neoplasia: a meta-analysis." International journal of preventive medicine $\mathbf{8}$.

Haghshenas, M. R., T. Mousavi, M. Moosazadeh and M. Afshari (2016). "Human papillomavirus and breast cancer in Iran: a meta-analysis." Iranian journal of basic medical sciences 19(3): 231.

Jeannin, P., T. Renno, L. Goetsch, I. Miconnet, J.-P. Aubry, Y. Delneste, N. Herbault, T. Baussant, G. Magistrelli and C. Soulas (2000). "OmpA targets dendritic cells, induces their maturation and delivers antigen into the MHC class I presentation pathway." Nature immunology. 1(6): 502.

Kim, C.-H., M.-J. Hong, S.-D. Park, C.-K. Kim, M.-Y. Park, H.-J. Sohn, H.-I. Cho, T.-G. Kim and Y.-K. Hong (2006). "Enhancement of anti-tumor immunity specific to murine glioma by vaccination with tumor cell lysate-pulsed dendritic cells engineered to produce interleukin-12." Cancer Immunology, Immunotherapy. 55(11): 1309-1319.

Kim, T. G., C. H. Kim, E. H. Won, S. M. Bae, W. S. Ahn, J. B. Park and J. I. Sin (2004). "CpG-ODN-stimulated dendritic cells act as a potent adjuvant for E7 protein delivery to induce antigen-specific antitumour immunity in a HPV 16 E7-associated animal tumour model." Immunology. 112(1): 117-125.

Laus, R., T. J. Graddis, I. Hakim and D. Vidovic (2000). "Enhanced major histocompatibility complex class I-dependent presentation of antigens modified with cationic and fusogenic peptides." Nature biotechnology 18(12): 1269.

Levy, O., S. Goriely and T. R. Kollmann (2013). "Immune response to vaccine adjuvants during the first year of life." Vaccine 31(21): 2500-2505.

Li, J., S. Chen, J. Ge, F. Lu, S. Ren, Z. Zhao, X. Pu, X. Chen, J. Sun and Y. Gu (2017). "A novel therapeutic vaccine composed of a rearranged human papillomavirus type $16 \mathrm{E} 6 / \mathrm{E} 7$ fusion protein and Fms-like tyrosine kinase-3 ligand induces CD8+ T cell responses and antitumor effect." Vaccine 35(47): 64596467.

Mellman, I. and R. M. Steinman (2001). "Dendritic cells: specialized and regulated antigen processing machines." Cell 106(3): 255-258.

Monroy-García, A., M. A. Gómez-Lim, B. Weiss-Steider, J. Hernández-Montes, S. Huerta-Yepez, J. F. RangelSantiago, E. Santiago-Osorio and M. d. L. M. García (2014). "Immunization with an HPV-16 L1-based chimeric virus-like particle containing HPV-16 E6 and E7 epitopes elicits long-lasting prophylactic and therapeutic efficacy in an HPV-16 tumor mice model." Archives of virology. 159(2): 291-305.

Mousavi, T., A. Rafiei, M. reza Haghshenas, S. F. Sadeghian-Kiadehi and R. Valadan (2020). "Molecular prevalence and phylogenetic analysis of human papillomavirus in normal cervical samples from northern Iran." Gene Reports: 100958. 
Mousavi, T., S. S. Saravi, R. Valadan, M. R. Haghshenas, A. Rafiei, H. Jafarpour and A. Shamshirian (2019). "Different types of adjuvants in prophylactic and therapeutic human papillomavirus vaccines in laboratory animals: a systematic review." Archives of Virology: 1-22.

Münger, K., E. J. Androphy and A. A. McBride (2018). Regulation of the Papillomavirus E6 and E7 Oncoproteins By the Viral E1 and E2 Proteins. Viral Regulatory Structures And Their Degeneracy, CRC Press: 35-52.

Shahbazi, S. and A. Bolhassani (2018). "Comparison of six cell penetrating peptides with different properties for in vitro and in vivo delivery of HPV16 E7 antigen in therapeutic vaccines." International Immunopharmacology 62: 170-180.

Singh-Jasuja, H., R. E. Toes, P. Spee, C. Münz, N. Hilf, S. P. Schoenberger, P. Ricciardi-Castagnoli, J. Neefjes, H.-G. Rammensee and D. Arnold-Schild (2000). "Cross-presentation of glycoprotein 96associated antigens on major histocompatibility complex class I molecules requires receptor-mediated endocytosis." Journal of Experimental Medicine 191(11): 1965-1974.

Su, X., W. Xu, R. Guan, Y. Wang, J. Wu, L. Zhai, G. Chen and S. Hu (2016). "Adjuvant effect of docetaxel on HPV16 L2E6E7 fusion protein vaccine in a mouse model." International immunopharmacology 38: 16-25.

Tanaka, Y., S. F. Dowdy, D. C. Linehan, T. J. Eberlein and P. S. Goedegebuure (2003). "Induction of antigenspecific CTL by recombinant HIV trans-activating fusion protein-pulsed human monocyte-derived dendritic cells." The Journal of Immunology_170(3): 1291-1298.

Tang, J., R. Yin, Y. Tian, Z. Huang, J. Shi, X. Fu, L. Wang, Y. Wu, F. Hao and B. Ni (2012). "A novel selfassembled nanoparticle vaccine with HIV-1 Tat49-57/HPV16 E749-57 fusion peptide and GM-CSF DNA elicits potent and prolonged CD8+ T cell-dependent anti-tumor immunity in mice." Vaccine 30(6): 10711082.

Wu, X., X. Ma, Y. Li, Y. Xu, N. Zheng, S. Xu, W. Nawaz and Z. Wu (2019). "Induction of neutralizing antibodies by human papillomavirus vaccine generated in mammalian cells." Antibody Therapeutics 2(2): 45-53.

Xu, W.-X., J. Wang, H.-P. Tang, Y.-P. He, Q.-X. Zhu, S. K. Gupta, S.-H. Gu, Q. Huang, C.-N. Ji and L.-F. Liu (2016). "Epitomics: IgG-epitome decoding of E6, E7 and L1 proteins from oncogenic human papillomavirus type 58." Scientific reports 6: 34686.

Zhao, B., Y. Wang, Y. Zhang, Y. Li, X. Zhang, Y. Xu, L. Chen, C. Li, Y. Ju and S. Meng (2013). "TAT-mediated gp96 transduction to APCs enhances gp96-induced antiviral and antitumor T cell responses." Vaccine 31(3): 545-552.

Zou, L., Q. Peng, P. Wang and B. Zhou (2017). "Progress in research and application of HIV-1 TAT-derived cell-penetrating peptide." The Journal of membrane biology 250(2): 115-122. 


\section{Note}

Figures and tables were not included with this version. 